\title{
Microscopy with ultraviolet surface excitation for wide-area pathology of breast surgical margins
}

Weisi Xie

Ye Chen

$\mathrm{Yu}$ Wang

Linpeng Wei

Chengbo Yin

Adam K. Glaser

Mark E. Fauver

Eric J. Seibel

Suzanne M. Dintzis

Joshua C. Vaughan

Nicholas P. Reder

Jonathan T. C. Liu 


\title{
Microscopy with ultraviolet surface excitation for wide-area pathology of breast surgical margins
}

\author{
Weisi Xie, ${ }^{\text {a }}$ Ye Chen, ${ }^{a}$ Yu Wang, ${ }^{\text {a }}$ Linpeng Wei, ${ }^{\text {a }}$ Chengbo Yin, ${ }^{a}$ Adam K. Glaser, ${ }^{a}$ Mark E. Fauver, ${ }^{a}$ \\ Eric J. Seibel, ${ }^{a}$ Suzanne M. Dintzis, ${ }^{b}$ Joshua C. Vaughan, ${ }^{c}$ Nicholas P. Reder, ${ }^{b}$ and Jonathan T. C. Liu ${ }^{a, b, *}$ \\ aniversity of Washington, Department of Mechanical Engineering, Seattle, Washington, United States \\ bUniversity of Washington, School of Medicine, Department of Pathology, Seattle, Washington, United States \\ 'University of Washington, Department of Chemistry, Seattle, Washington, United States
}

\begin{abstract}
Intraoperative assessment of breast surgical margins will be of value for reducing the rate of re-excision surgeries for lumpectomy patients. While frozen-section histology is used for intraoperative guidance of certain cancers, it provides limited sampling of the margin surface (typically $<1 \%$ of the margin) and is inferior to gold-standard histology, especially for fatty tissues that do not freeze well, such as breast specimens. Microscopy with ultraviolet surface excitation (MUSE) is a nondestructive superficial optical-sectioning technique that has the potential to enable rapid, high-resolution examination of excised margin surfaces. Here, a MUSE system is developed with fully automated sample translation to image fresh tissue surfaces over large areas and at multiple levels of defocus, at a rate of $\sim 5 \mathrm{~min} / \mathrm{cm}^{2}$. Surface extraction is used to improve the comprehensiveness of surface imaging, and 3-D deconvolution is used to improve resolution and contrast. In addition, an improved fluorescent analog of conventional H\&E staining is developed to label fresh tissues within $\sim 5$ min for MUSE imaging. We compare the image quality of our MUSE system with both frozen-section and conventional H\&E histology, demonstrating the feasibility to provide microscopic visualization of breast margin surfaces at speeds that are relevant for intraoperative use. $\odot$ The Authors. Published by SPIE under a Creative Commons Attribution 4.0 Unported License. Distribution or reproduction of this work in whole or in part requires full attribution of the original publication, including its DOI. [DOI: 10.1117/1 .JBO.24.2.026501]

Keywords: microscopy with UV surface excitation; 3-D deconvolution; fluorescence; H\&E staining; lumpectomy; surgical margin assessment.
\end{abstract}

Paper 180646R received Dec. 1, 2018; accepted for publication Jan. 21, 2019; published online Feb. 8, 2019.

\section{Introduction}

Lumpectomy is a breast-conserving procedure used to surgically remove breast carcinoma along with a margin of normal tissue surrounding the tumor. Over the years, studies have shown that patients with invasive breast cancer, treated with lumpectomy and local breast irradiation, exhibit no significant difference in survival compared with those undergoing mastectomy, provided that the entire tumor is removed during lumpectomy surgery. ${ }^{1,2}$ However, if postoperative histopathology reveals the presence of carcinoma at the surgical margin, re-excision procedures, including completion mastectomies, are often performed to minimize the possibility of local recurrence. ${ }^{3}$ Reoperation after lumpectomy can result in the delay of adjuvant treatments with increased risks of recurrence, ${ }^{4}$ emotional trauma, ${ }^{5}$ as well as an increased economic burden for both the healthcare system and patients. The rate of reoperation after lumpectomy has been calculated to be at least $20 \%$ according to recent studies. ${ }^{3,6,7}$ Therefore, there is an acknowledged need for reliable intraoperative methods to assess the surgical margins of freshly resected lumpectomy specimens (Fig. 1).

Conventional gold-standard histopathology relies upon the microscopic imaging of thin tissue sections (usually $\sim 4-\mu \mathrm{m}$ thick) mounted on glass slides and stained with hematoxylin and eosin. This lengthy process of tissue fixation, dehydration, paraffin embedding, sectioning, and staining requires hours to days and is not possible within intraoperative time frames. Additionally, only a few thin cross sections of the sample are

*Address all correspondence to Jonathan T. C. Liu, E-mail: jonliu@uw.edu examined in conventional histology, where there is extremely limited sampling of the surgical margin surface. ${ }^{8}$ As an alternative, frozen-section histology enables rapid intraoperative assessments to guide certain resection procedures, ${ }^{9}$ but suffers from inferior image quality and similar sampling limitations as standard histology. ${ }^{10,11}$ Furthermore, frozen sections require the destructive removal of tissues that should ideally be preserved for gold-standard postoperative histology. Finally, frozen sectioning introduces freezing artifacts, especially in fatty breast tissues, which negatively impact the quality of downstream postoperative histology. ${ }^{12}$

Optical-sectioning microscopy techniques can potentially enable rapid nondestructive imaging of surgical margin surfaces to guide tumor-resection procedures. For instance, confocal microscopy, ${ }^{13-15}$ nonlinear microscopy, ${ }^{16-20}$ open-top lightsheet (OTLS) microscopy, ${ }^{21}$ structured-illumination microscopy (SIM) ${ }^{22-24}$ photoacoustic microscopy (PAM), ${ }^{25}$ and optical coherence tomography (OCT), ${ }^{26,27}$ among others, have been shown to enable high-resolution imaging of freshly excised surgical specimens that can approach the quality of gold-standard histology. All of these techniques involve trade-offs between speed, simplicity, cost, resolution, and image contrast/depth. In particular, while laser-scanned microscopy techniques such as confocal and nonlinear microscopy can provide exquisite resolution and contrast/depth, even without exogenous contrast agents in the case of nonlinear microscopy, they tend to be more complex, bulky, slow, and expensive. For surface microscopy within a surgical setting, camera-based optical-sectioning techniques such as OTLS microscopy and SIM have clear benefits regarding speed, and in the case of SIM, clear advantages 


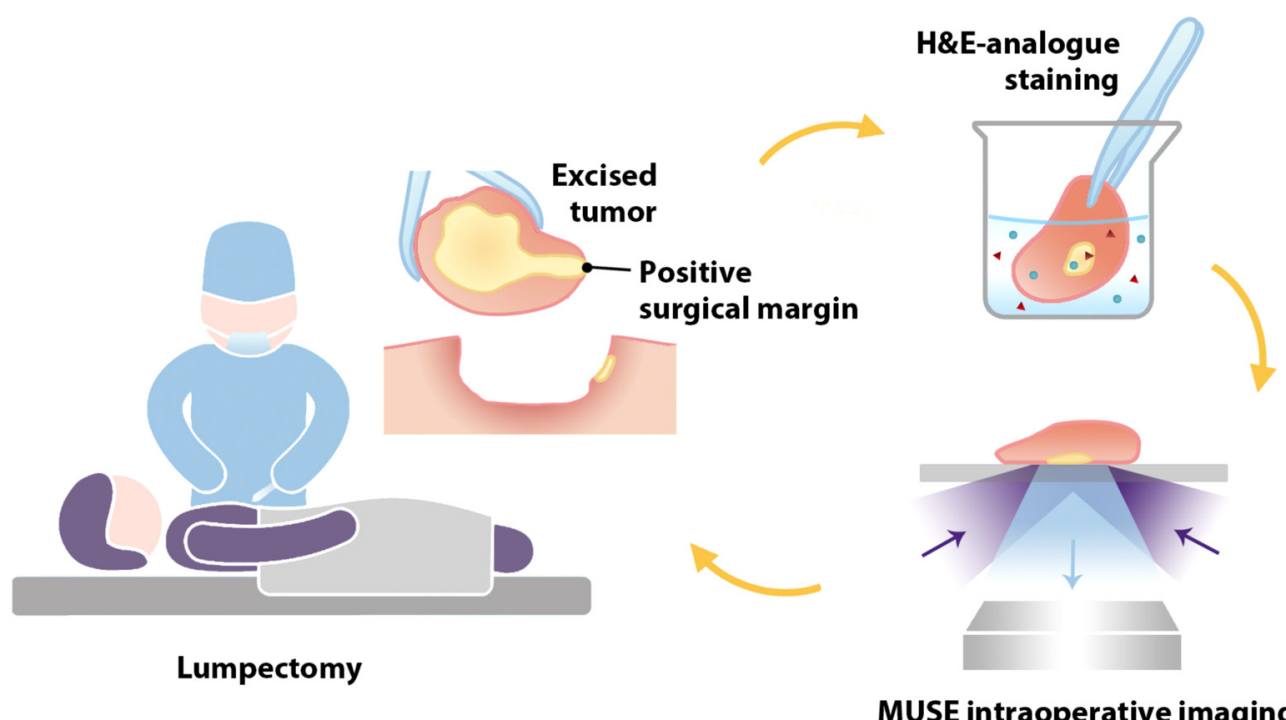

Fig. 1 Clinical workflow for intraoperative surgical margin assessment by MUSE. During lumpectomy, the freshly resected tissue specimen is immediately stained with a fluorescent analog of H\&E staining. The surgical margin surface of the resected specimen is then imaged with MUSE to guide the resection procedure.

concerning cost and simplicity. Finally, PAM and OCT can provide label-free high-resolution imaging based on absorption and reflectance contrast, respectively, but are somewhat limited in their ability to provide multicolor imaging of tissue structures that pathologists are accustomed to viewing (e.g., H\&E histology).

Here we explore a recent camera-based superficial opticalsectioning strategy, known as microscopy with ultraviolet surface excitation (MUSE), which is relatively simple, low cost, and potentially well-suited for intraoperative surgical margin assessment. ${ }^{28-30}$ With MUSE, the use of short-wavelength $(285 \mathrm{~nm})$ ultraviolet (UV) light limits the penetration of the illumination light to within $\sim 10-\mu$ m beneath the tissue surface, such that a relatively high-contrast "optically sectioned" image of the tissue surface may be obtained rapidly with a 2-D detector array. Many conventional visible-excited fluorescent dyes can be excited with UV illumination, with high-fluorescence yields in the visible range. ${ }^{28}$ Since the collected fluorescence is in the visible range, there is no need for expensive UV-compatible optics. Inexpensive UV LEDs may be used, rather than lasers, to generate sufficient illumination light over a reasonable field-ofview (typically on the millimeter scale). These features make MUSE particularly cost-effective as compared with other microscopy alternatives.

A recent study on MUSE $^{29}$ reported that the penetration depth of UV light (i.e., the optical-sectioning thickness) in tissues is $\sim 10$ to $20 \mu \mathrm{m}$, which is significantly thicker than conventional histology sections $(\sim 4 \mu \mathrm{m})$. Thicker sectioning degrades the image quality by introducing more background from out-of-focus light generated above and below the focal plane, which ultimately leads to a reduction in resolution and contrast. $^{31,32}$ To further approach the image quality of goldstandard histology, we collected a $z$ stack of MUSE images at each lateral position and applied 3-D deconvolution to improve the resolution and contrast. In addition, a surface extraction algorithm was used to minimize the effects of surface irregularities. With 3-D deconvolution, the point spread function
(PSF) of the imaging system is used to restore the detected image, including the resolution and signal-to-background ratio, such that the image provides a more faithful representation of the original object. ${ }^{32}$

To generate images that mimic the appearance of gold-standard $\mathrm{H} \& \mathrm{E}$ histology, which pathologists are accustomed to viewing, a two-color UV-excited fluorescent analog of H\&E staining was developed to rapidly stain fresh tissues for MUSE. This method utilizes SYBR Gold to stain cellular nuclei and ATTO 655 NHS ester to stain stromal/cytoplasmic structures. While eosin is naturally fluorescent and has previously been utilized in fluorescent analogs of H\&E staining, it does not bind well to fresh tissues and has often exhibited a "leakage" issue in tissues that are not formalin fixed and dehydrated. ${ }^{29,33}$ We show here that NHS esters can serve as an alternative to eosin for staining fresh, hydrated tissues with improved contrast and microscopic detail in comparison to eosin. Regarding speed, it is possible to effectively stain and rinse breast specimens within $\sim 5 \mathrm{~min}$ followed by MUSE imaging at a rate of $\sim 5 \mathrm{~min} / \mathrm{cm}^{2}$. We show examples to demonstrate that the image quality of our MUSE approach surpasses that of frozen-section histology and also compare the image quality of MUSE with archival H\&E histology of formalin-fixed paraffin-embedded (FFPE) tissues. Specific differences in appearance will be highlighted as we evaluate the feasibility of this approach for intraoperative surgical margin assessment to guide lumpectomy procedures.

\section{Methods}

\subsection{Collection and Preparation of Mouse Kidney}

Mouse kidneys were freshly excised from euthanized mice obtained through the animal donation program at the University of Washington. The kidneys were bisected, and the cut surfaces were immediately stained and imaged with MUSE. 


\subsection{Collection and Preparation of Human Breast Tissue}

Excess fresh human breast tissues, destined for discard, were obtained from lumpectomies and mastectomies through the Northwest BioTrust (NWBT) at the University of Washington. The deidentified tissues were obtained with patient consent. Tissue surfaces were stained and imaged with MUSE. The tissues were then fixed in $10 \%$ formalin for 18 to $24 \mathrm{~h}$ and submitted for slide-based FFPE H\&E histology in $70 \%$ ethanol.

\subsection{UV-Excited Two-Color Fluorescent Analog of H\&E Staining for Fresh Tissue}

SYBR Gold $\left(\lambda_{\mathrm{Em}}=537 \mathrm{~nm}\right)$ was used as a nuclear stain, ${ }^{34}$ and ATTO $655 \mathrm{NHS}$ ester $\left(\lambda_{\mathrm{Em}}=680 \mathrm{~nm}\right)$ as a stromal/cytoplasmic stain [Fig. 2(a)]. Both of these fluorescent agents can be excited at $285 \mathrm{~nm}$. In our optimized protocol, fresh tissues were stained by submerging them in a $0.825: 10,000 \mathrm{v} / \mathrm{v}$ solution of SYBR Gold (Thermo Fisher, Cat. No: S11494) and 11.25- $\mu \mathrm{M}$ ATTO 655 NHS ester (Sigma-Aldrich) in $1 \times$ phosphate-buffered saline (PBS, Gibco, Cat. No: 10010023) at pH 8.0 for 5 min. The stained tissue was then rinsed 3 times in a large volume of $1 \times$ PBS ( $\mathrm{pH} 7.4$ ) for $30 \mathrm{~s}$ per wash, followed by MUSE imaging.

For control experiments to compare cytoplasmic/stromal staining approaches, a published MUSE protocol with eosin staining was used. $^{29}$ In short, tissues were stained with $200 \mu \mathrm{g} / \mathrm{ml}$ eosin for $2 \mathrm{~min}$ and then rinsed in $1 \times$ PBS.

\subsection{MUSE System Optimized for Dual-Channel Fluorescence Imaging}

An optical schematic of our MUSE system is shown in Fig. 2(b). UV illumination is provided by two 285-nm LEDs (M285L5,

(a)
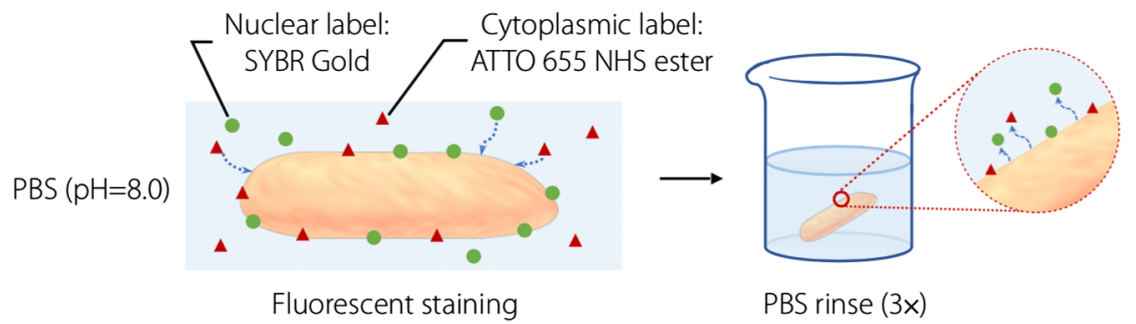

PBS rinse $(3 x)$

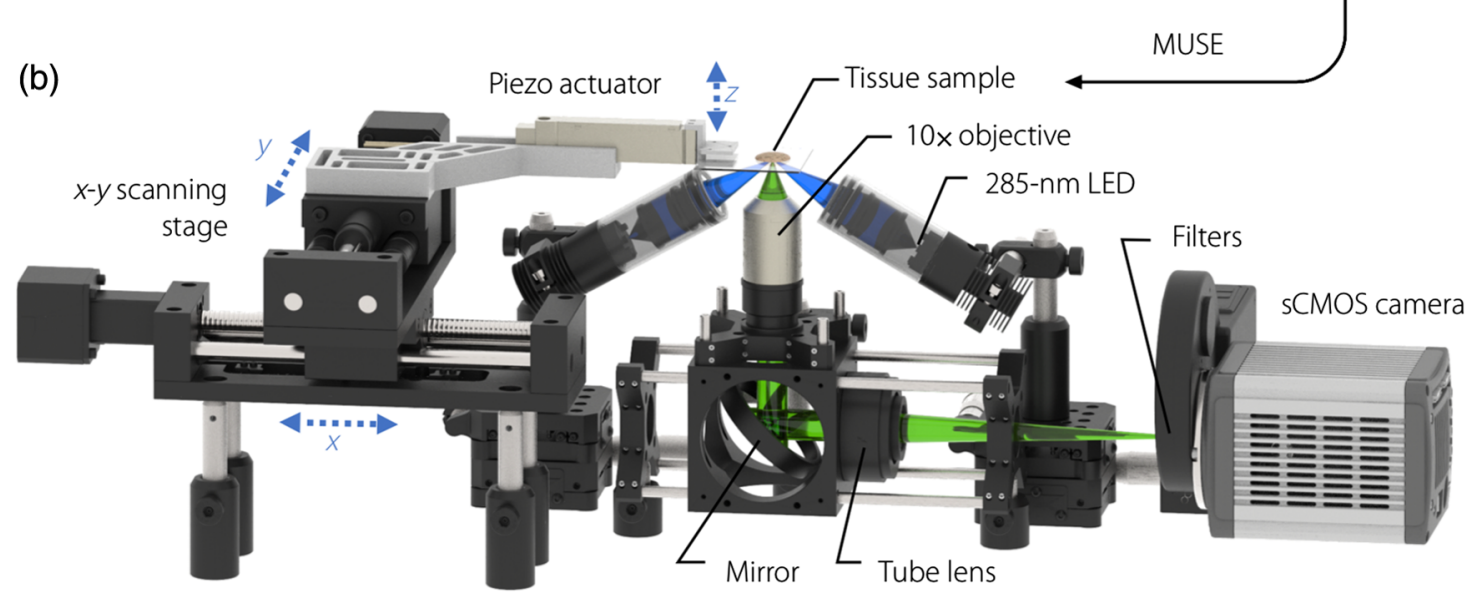

(c)
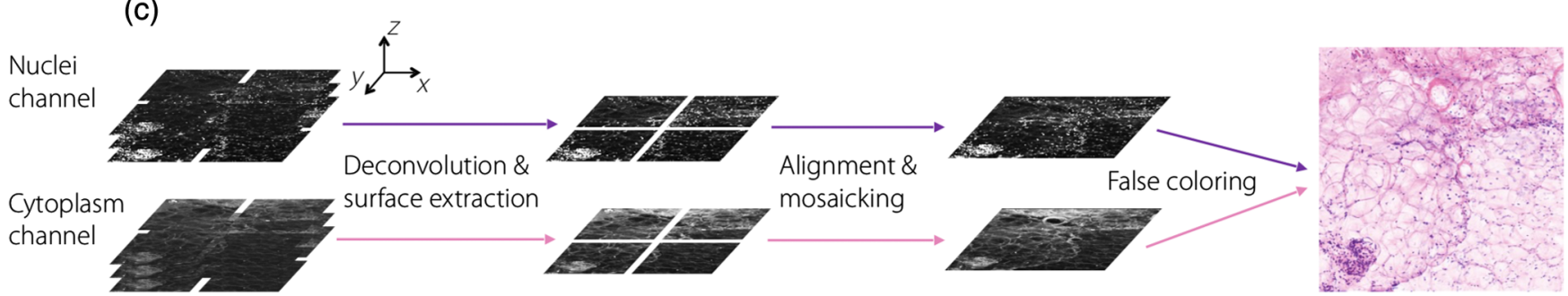

Fig. 2 Schematic of intraoperative MUSE system. (a) A fluorescent analog of H\&E staining is used to label fresh tissues within $\sim 5$ min. (b) A MUSE system for comprehensive imaging of fresh specimens. Two LEDs (285-nm wavelength) illuminate the specimen surface at an oblique angle from opposite directions to reduce shadowing artifacts. Fluorescence signal (visible wavelength) is collected and imaged by a $10 \times(N A=0.3)$ apochromatic objective and tube lens onto a 2-D detector array. A filter wheel is used to image the two-color channels sequentially. Large-area tiled imaging is achieved by scanning the specimen with a motorized $X Y$ stage. (c) At each imaged location (lateral image tile), the piezoactuator scans the specimen vertically to obtain a $z$ stack of images, which allows for 3-D deconvolution (to improve resolution and contrast), surface extraction (to mitigate the effects of surface irregularities), and false coloring to mimic the appearance of gold-standard H\&E histology. 
Thorlabs), in which the light is roughly collimated and focused at the specimen through the use of a pair of identical UV-fusedsilica lenses ( $F=35.0 \mathrm{~mm}$, LA4052-UV Thorlabs). The two UV beams pass through the clean-up filters (FF-1-285/14-25, Semrock) and illuminate the tissue surface at an oblique angle (to avoid being clipped by the large collection objective) from opposite directions to reduce shadowing artifacts. The sample is placed on a UV-fused-silica slide, in which the bottom surface of the tissue sample (illuminated surface) is positioned slightly below the focal plane of the UV beam so that the illumination spot is enlarged and generates a relatively uniform irradiance distribution across the field-of-view of the collection objective, with an average power of $14 \mathrm{~mW}$ (power density $\sim 1.55 \times 10^{3} \mathrm{~W} \mathrm{~m}^{-2}$ ) incident on the sample. For large-area imaging of tissue specimens, ultrasound gel (Parker Laboratories, Inc.) was placed around the edge of the specimen to reduce tissue deformation caused by dehydration during imaging and to obtain a clearer image of the tissue edge (due to index matching through the gel, rather than air). Visible-wavelength fluorescence signal is collected and imaged by a $10 \times$ apochromatic objective (CFI Plan Fluor $10 \times, \mathrm{NA}=0.3$, Nikon) and tube lens (ITL200, Thorlabs) onto a 2-D detector array (ORCA-Flash4.0 V3 Digital CMOS camera, Hamamatsu) with a lateral resolution of $\sim 1 \mu \mathrm{m}$ and a fieldof-view of $1.3 \mathrm{~mm} \times 1.3 \mathrm{~mm}$. The axial resolution of the 0.3 -NA objective is $\sim 12 \mu \mathrm{m}$, which roughly matches the penetration depth of UV light in fresh breast, which is about 10 to $20 \mu \mathrm{m}$. Large-area tiled imaging is achieved by scanning the specimen with a motorized $X Y$ stage (ET-50-11, Newmark systems). At each lateral imaging location, the piezoactuator (P-601.4SL, PI) scans the specimen vertically twice to obtain two $z$ stacks of images, in which a motorized filter wheel is used to change the optical filters of 550/40 BP (FB550-40, Thorlabs) for the nuclei channel and 664 LP (LP02-664RU-25, Semrock) for the cytoplasm channel between the two vertical scans. This rapid collection of the two image channels prevents channel co-registration problems due to tissue deformation over time.

\subsection{Image Acquisition and Postprocessing}

Image tiles are collected at a lateral spacing of $1 \mathrm{~mm}$ in both the $x$ and $y$ directions, which represents an optimal balance between imaging speed and mosaicking accuracy. The vertical step size is $5 \mu \mathrm{m}$, which satisfies the Nyquist sampling criterion (assuming an axial resolution of $\sim 12 \mu \mathrm{m}$ ). The total $z$-scanning range is $150 \mu \mathrm{m}$, which is sufficient to accommodate a majority of the surface irregularities seen at the tissue surface. The integration time for each frame is $50 \mathrm{~ms}$ (UV radiant exposure per frame of $\sim 77.5 \mathrm{~J} \mathrm{~m}^{-2}$ ), which represents a balance between speed and signal-to-noise ratio (SNR). Large-area image acquisitions are automatically controlled by a custom-developed LabVIEW program.

Data postprocessing consists of the following steps [Fig. 2(c)]: (1) 3-D deconvolution is performed for contrast and resolution enhancement. Ten iterations (optimized for speed and image quality) of a Lucy-Richardson algorithm (MATLAB) are applied for each vertical image stack ( $z$ stack). (2) Surface extraction is performed for surface-irregularity mitigation. For each $z$ stack of deconvolved images, we used an open source ImageJ plugin to perform a complex wavelet transform ${ }^{35}$ that takes a vertical stack of images and extracts the best focus (tissue surface) for each lateral subregion (a)

$$
\text { PSF measurement }
$$

(b)

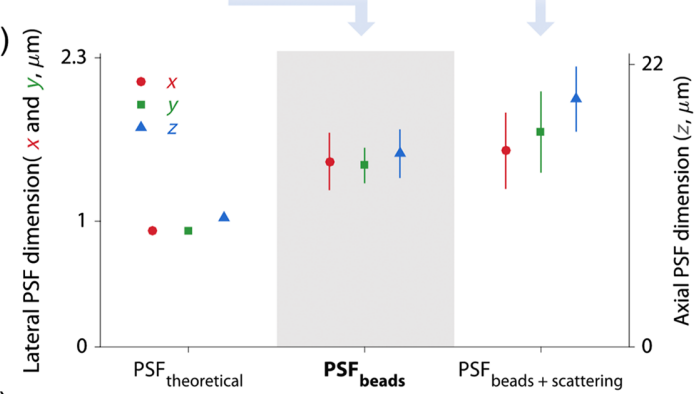

(c)
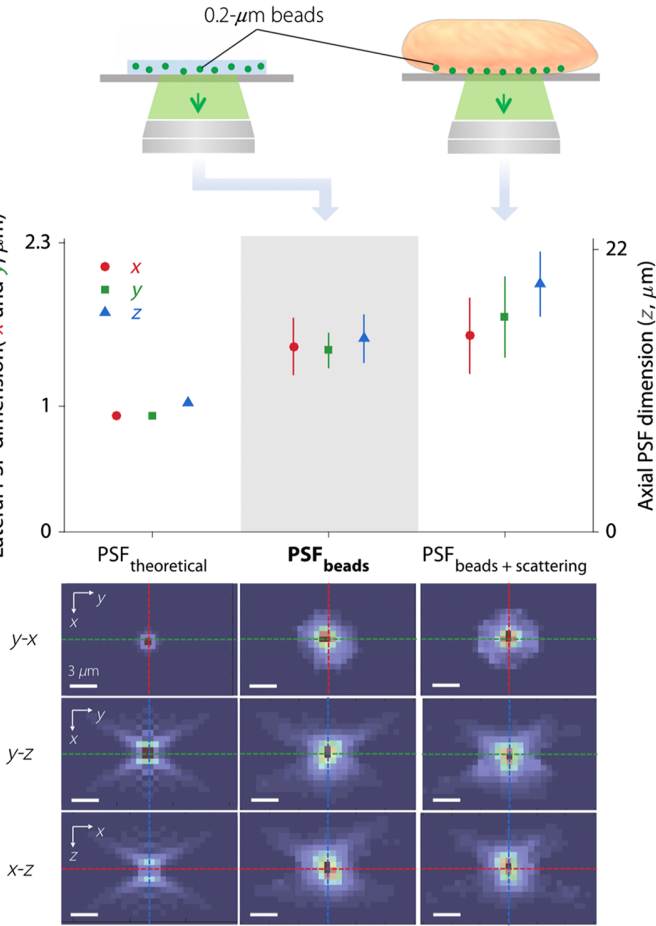

(d)

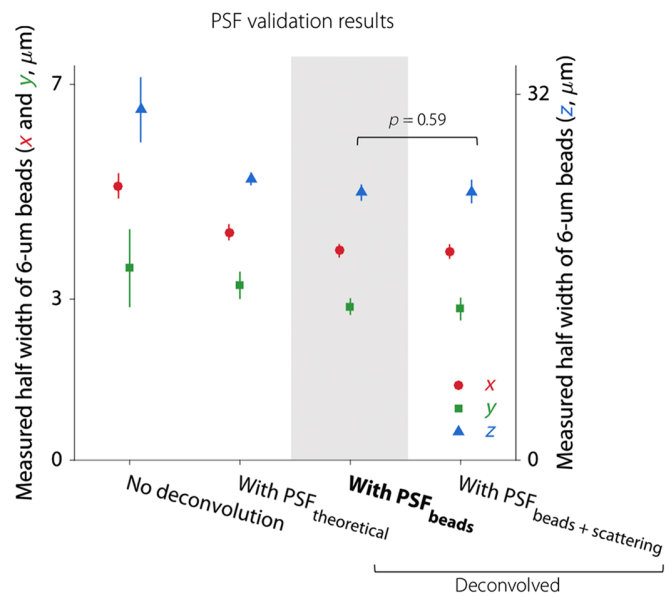

Fig. 3 Obtaining and validating a PSF for deconvolution. (a) Illustration of two PSF measurement methods, one of which images subresolution fluorescent beads $(d=\sim 0.2 \mu \mathrm{m}$, emission peak at $520 \mathrm{~nm}$ ) in an agarose phantom (standard method) and another of which images the same beads at the surface of human breast tissue (which adds the effects of tissue scattering). (b) Results showing the FWHM of the PSF theoretically calculated from system parameters $\left(\mathrm{PSF}_{\text {theoretical }}\right)$, the average PSF experimentally measured with beads in an agarose phantom $\left(\mathrm{PSF}_{\text {beads }}\right)$, and the average PSF experimentally measured with beads at the tissue surface $\left(\mathrm{PSF}_{\text {beads+scattering }}\right)$. (c) Three orthogonal cross-sectional views of $\mathrm{PSF}_{\text {theoretical }}, \mathrm{PSF}_{\text {beads }}$, and $\mathrm{PSF}_{\text {beads+scattering. The colors }}$ of the dashed lines correspond to the colors of the data points in (b) and (d) for the $x, y$, and $z$ directions (red, green, and blue, respectively). Scale bar: $3 \mu \mathrm{m}$. (d) PSF validation results showing the average FWHM of 6- $\mu \mathrm{m}$ beads at a tissue surface without deconvolution, and with deconvolution using three different PSFs. The use of $\mathrm{PSF}_{\text {beads }}$ for deconvolution yields the best results regarding

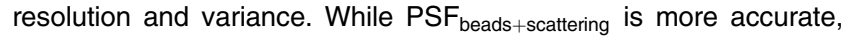
it does not provide improved deconvolution results (as shown by the $p$-value). (b) and (d) Left vertical axis: FWHM in the $x$ and $y$ directions (red and green). Right vertical axis: FWHM in $z$ direction (blue). 
(the parameters for the algorithm are tunable and provide a trade-off between extraction quality and speed). (3) The images from the two channels at each lateral position are co-registered (aligned) with a normalized cross-correlation algorithm. (4) The aligned images for each channel are mosaicked using the grid/collection stitching plugin in ImageJ. ${ }^{36}$ (5) The large-area (mosaicked) images are falsecolored (by combining the two image channels) to mimic the appearance of gold-standard H\&E histology. Here we have optimized an $H \& E$ false-coloring algorithm that was previously published. ${ }^{37}$ In short, localized histogram equalization is applied to each channel, prior to $\mathrm{H} \& \mathrm{E}$ false coloring, to enhance the contrast and consistency of the false coloring across a large-area image.

\subsection{Statistical Analysis and Quantitative Evaluation of Image Quality}

The data points shown in Fig. 3 present mean values from multiple measurements, and the error bars show the range of measured data. Statistical significance was calculated by a $t$-test (two-sample, assuming unequal variances) for $\mathrm{FWHM}_{x} *$ $\mathrm{FWHM}_{y} * \mathrm{FWHM}_{z}$ of the $6-\mu \mathrm{m}$ beads deconvolved by $\mathrm{PSF}_{\text {beads }}$ and $\mathrm{PSF}_{\text {beads+scattering. The level of significance was set at }}$ $P<0.05$.

In Fig. 4, contrast is calculated by $\left(I_{\max }-I_{\min }\right) /$ $\left(I_{\max }+I_{\min }\right)$ from the line profiles, where $I_{\max }$ and $I_{\min }$ represent the maximum and minimum intensities, respectively. Contrast-to-noise ratio (CNR) is calculated by $\left(I_{\max }-I_{\min }\right) /$

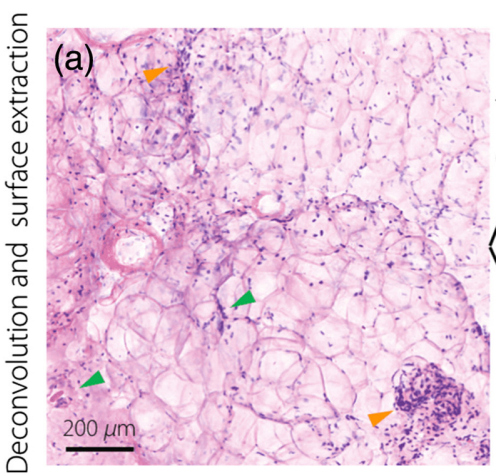

Nuclear channel, region 1
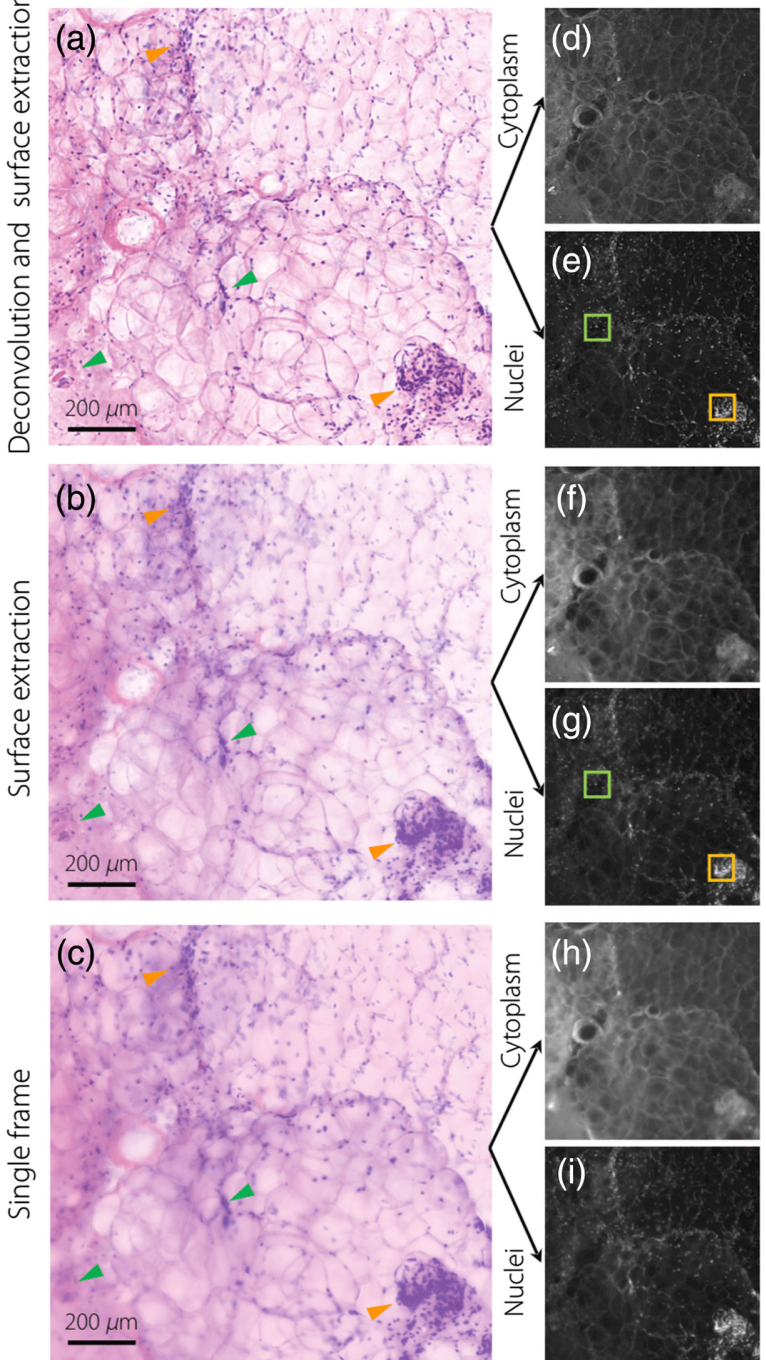
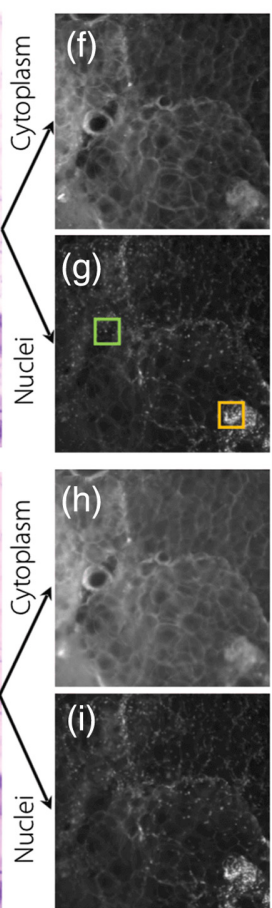
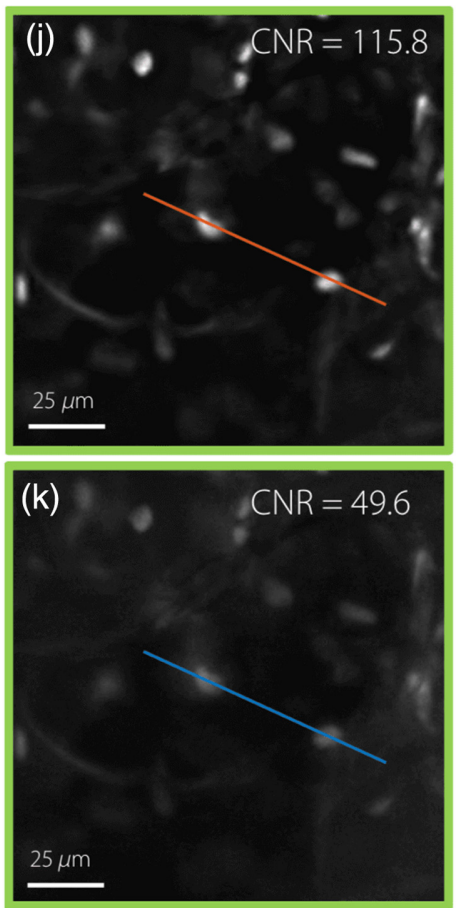

(n)

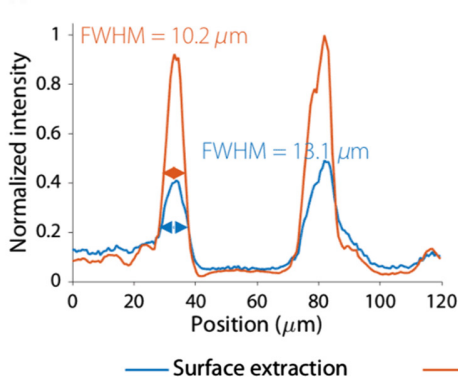

Nuclear channel, region 2
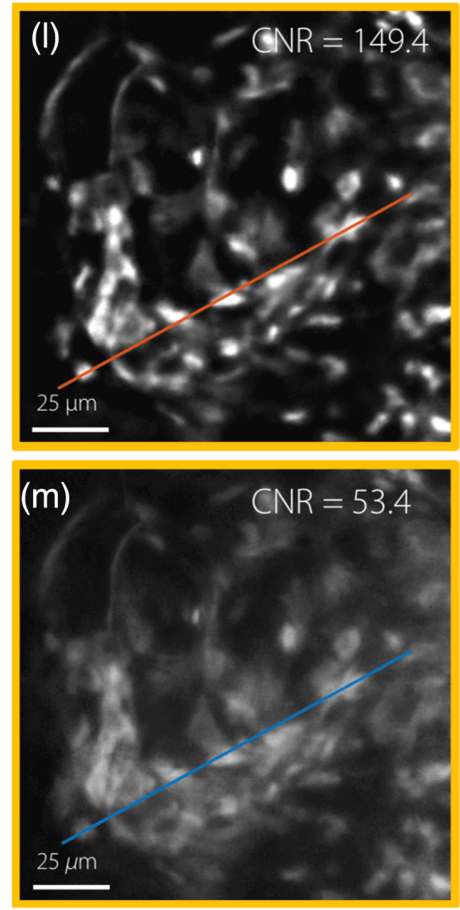

(o)

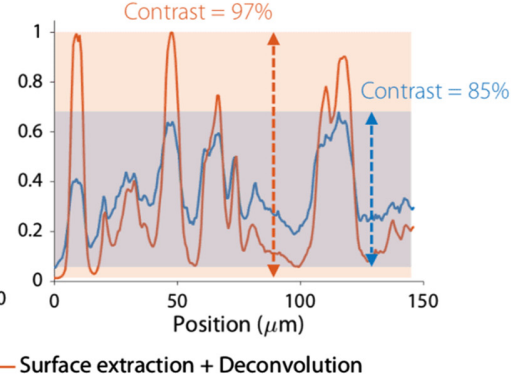

Fig. 4 A comparison of image quality for MUSE of breast specimens. Results are shown (a) with deconvolution and surface extraction, (b) with only surface extraction, and (c) of a single frame within a $z$ stack. The green arrows indicate features that are out of focus in a single frame but are brought into focus by surface extraction. The orange arrow points to a cluster of nuclei that are significantly more resolved after deconvolution. These MUSE images are false-colored to mimic H\&E histology, based on (e), (g), and (i) a nuclear channel and (d), (f), and (h) a stromal/cytoplasmic channel. (j)-(m) Two zoomed-in regions from the nuclear channel show that deconvolution enables the resolution, contrast, and CNR to be improved. (n) A line profile from region 1 shows that the FWHM of the nuclei are smaller (more resolved) with deconvolution than without. (o) A line profile from region 2 shows that the overall image contrast is also improved by deconvolution. 
noise from the images, where noise denotes the standard deviation of the intensity in background region of the images.

\section{Results and Discussion}

Measurements and validation of the PSF of our MUSE system are shown in Fig. 3. All of the experimental data shown in the diagrams are averaged from 8 to 10 samples with the indicated uncertainties (standard deviation). In the PSF measurement results shown in Fig. 3(b), both of the experimentally measured PSFs ( $\mathrm{PSF}_{\text {beads }}$ and $\mathrm{PSF}_{\text {beads+scattering }}$ ) show a slightly larger fullwidth at half-maximum (FWHM) than the theoretically calculated PSF ( $\left.\mathrm{PSF}_{\text {theoretical }}\right)$. This is due to a variety of minor aberrations introduced by the experimental system. For the two experimental PSFs, the FWHM of PSF $_{\text {beads+scattering is slightly }}$ larger than $\mathrm{PSF}_{\text {beads }}$. This is expected since beads located at the surface of a scattering tissue (human breast in this study) are influenced by the scattering background, which leads to a slightly enlarged PSF. Theoretically, among the three PSFs we obtained, PSF $_{\text {beads+scattering }}$ should most accurately describe the blurring of point objects in tissues that are imaged with MUSE (i.e., due to diffraction, aberrations, and tissue-scattering background). For more intuitive visual interpretation of Fig. 3(b), three orthogonal views of the measured PSFs are shown in Fig. 3(c).

To determine which PSF to use for MUSE image deconvolution, images of $6-\mu \mathrm{m}$ beads at breast tissue surfaces were deconvolved using the three PSFs described above (experimentally averaged PSFs). The FWHM of the $6-\mu \mathrm{m}$ beads before and after deconvolution is shown in Fig. 3(d). The asymmetry in the size of beads in the $x$ and $y$ directions is because the two-sided illumination employed in our system is aligned along the $x$ direction [Fig. 2(b)]. The experimental PSFs generate the best improvements in resolution through deconvolution (compared to the theoretical PSF). However, no significant difference is observed between the dimensions of the $6-\mu \mathrm{m}$ beads deconvolved with the two different experimental PSFs, as shown by the $p$-value. Furthermore, the FWHM of the beads deconvolved with $\mathrm{PSF}_{\text {beads }}$ exhibits less variability than that using

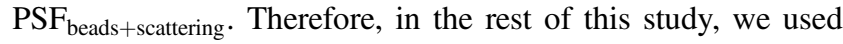
$\mathrm{PSF}_{\text {beads }}$ for all deconvolution procedures.

Figure 4 shows an image-quality comparison for breast tissues imaged with MUSE. Examples are shown in which deconvolution and surface extraction are both used, surface extraction is used alone, and a single unprocessed frame within a $z$ stack. Surface extraction of a $z$ stack (150- $\mu$ m range) brings a number of out-of-focus features (caused by surface irregularities) into focus within a single-frame image, as indicated by the green arrows. Deconvolution further improves the image resolution, contrast, and CNR, as shown in the zoomed-in nuclear-channel images [Figs. 4(j)-4(m)] and line profiles [Figs. 4(n) and 4(o)]. In summary, the use of 3-D deconvolution allows features to be better resolved, as indicated by the yellow arrows in Figs. 4(a)-4(c).

To demonstrate that ATTO 655 NHS ester can serve as a superior stromal/cytoplasmic contrast agent for fresh tissue in comparison to eosin for a two-color fluorescent analog of H\&E, MUSE images of mouse kidney stained with ATTO 655 NHS ester and eosin are shown in Fig. 5. As a result of improved tissue binding, the ATTO 655 NHS ester can reduce the pooling effects caused by eosin leakage and also provides an enhancement in microstructural contrast.

The image quality of MUSE versus frozen sectioning is shown in Fig. 6. In the frozen sections, freezing artifacts results

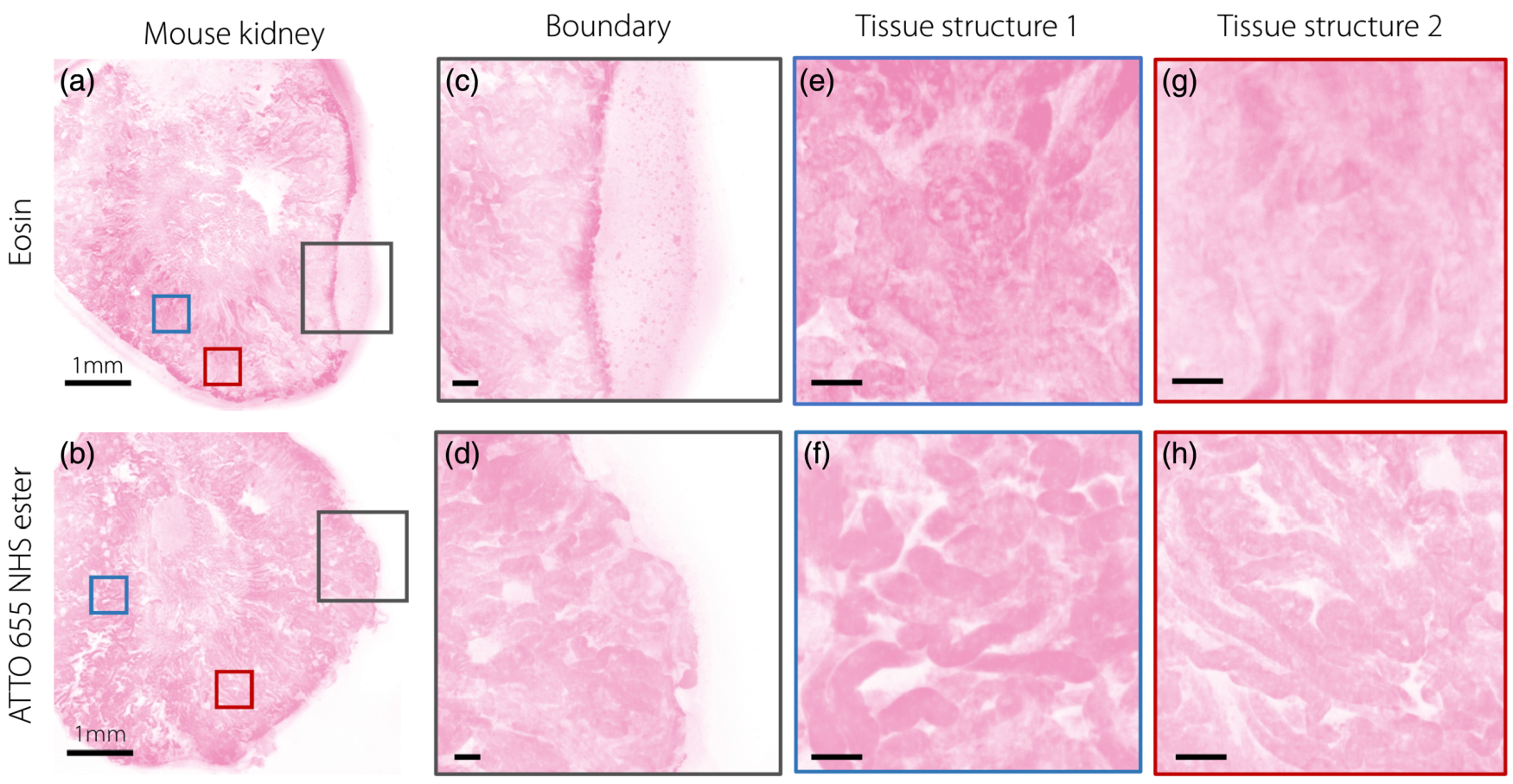

Fig. 5 A comparison of MUSE image quality with various stromal/cytoplasmic stains. The images show examples of fresh tissues stained with (a) eosin and (b) ATTO 655 NHS ester. (c) With fresh, hydrated specimens, the leakage of eosin, which is poorly bound, results in a pooling effect around the edge of the specimen, and also reduces (e) and (g) image contrast of microstructures. ATTO 655 NHS ester does not exhibit leaking at the $(d)$ tissue edges and provides improved $(f)$ and $(h)$ microstructural contrast. Scale bar: (a) and (b) $1 \mathrm{~mm}$ and (c)-(h) $100 \mu \mathrm{m}$. 

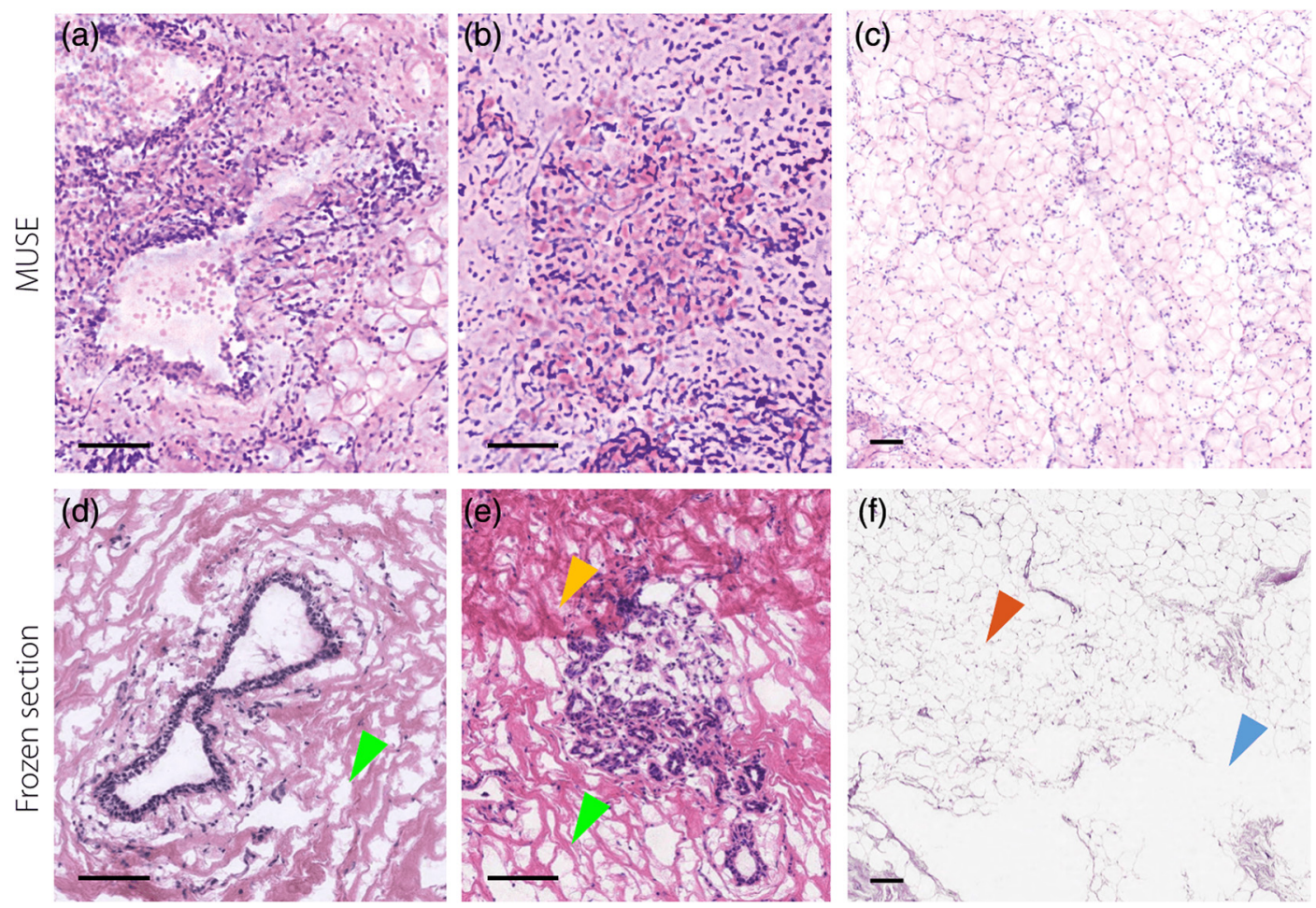

Fig. 6 Human breast tissue imaged with MUSE in comparison to frozen section histology. Large ducts are shown in (a) MUSE and (d) frozen-section histology images. (d) and (e) The frozen sections exhibit optically clear spaces, as indicated by the green arrow) between collagen fibers, which are freezing artifacts that do not appear in the MUSE images. Lobular units are shown in (b) MUSE and (e) frozen section histology images. The yellow arrow in (e) points to a tissue-fold artifact in the frozen section. Adipose tissue is shown in (c) MUSE and (f) frozen-section histology images. The red arrow in (f) indicates distorted adipose tissue and the blue arrow indicates a tear artifact from sectioning the frozen breast tissue. Scale bar: $100 \mu \mathrm{m}$.

in uneven stromal structure with looser collagen [Figs. 6(d) and 6(e), green arrow] and distortion of the lipids [Fig. 6(f), red arrow]. In particular, the adipocytes are highly disrupted and poorly sectioned in the frozen sections [Fig. 6(f), blue arrow]. Tissue folds are also apparent [Fig. 6(e), yellow arrow], which can potentially obscure underlying regions of carcinoma. These diagnosis-impairing issues in the frozen sections are not present in the MUSE images because the tissues are imaged fresh and without physical sectioning.

Figures 7(e)-7(j), 8(a), and 8(b) show that our customized MUSE system can enable comprehensive pathology of largearea freshly excised breast specimen surfaces. Image quality is compared to that of gold-standard slide-based FFPE H\&E histology. The zoomed-in images and insets show benign breast lobules [Figs. 7(d) and 7(e)], stroma and neurovascular bundles [Figs. 7(f) and 7(g)], adipose tissue [Figs. 8(e)-8(g)], a benign duct [Figs. 8(h)-8(j)], and ductal carcinoma in situ (DCIS) [Figs. 8(k)-8(m)].

Differences exist between MUSE and slide-based FFPE H\&E images. For example, the NHS ester staining for MUSE appears to be darker and more spatially distributed than the eosin staining for slide-based FFPE histology; cross sections of individual acini often show optically clear lumens in slidebased FFPE H\&E images [Fig. 7(e)], whereas the acini shown in the MUSE images are filled with eosinophilic material [Fig. 7(d)]; zoomed-in views of the vein [Fig. 7(f)] and duct [Fig. 8(h)] in the MUSE image show an oblique perspective with a short segment of the inner walls, which are absent in slide-based FFPE H\&E images [Figs. 7(g) and 8(i)]. This oblique perspective is discussed further in the next few paragraphs. Multiple layers of adipocytes are seen in the MUSE images [Fig. 8(e)] while only a monolayer of cross-sectioned adipocytes is viewed against a clean background in slidebased FFPE H\&E images [Fig. 8(f)]. The epithelial cells filling the lumen of the duct appear less basophilic in the MUSE image [Fig. 8(k)] than in the slide-based FFPE H\&E image [Fig. 8(1)].

We speculate that the differences in image quality between MUSE and conventional histology are due to three main reasons. First, as stated for the staining comparison in Fig. 5, ATTO 655 NHS ester binds covalently to cytoplasmic proteins, with improved tissue binding than eosin. Second, the optical sectioning thickness of MUSE for fresh breast tissues is on the order of 10 to $20 \mu \mathrm{m},{ }^{29}$ which is significantly larger than the thickness of a histology section $(\sim 4 \mu \mathrm{m})$. This increased section thickness of MUSE will result in increased background, which may obscure specific finer structures that are seen in the conventional histology sections. Finally, the surface topology and cellular contents that are present at the surfaces of grossly cut fresh tissue may be different from microtomesectioned FFPE tissues. For example, the acini/cells may be cut open during microtome sectioning of FFPE tissues while the surfaces of grossly cut fresh tissue may not contain intraacinar/intracellular contents. In addition, grossly cut tissue may exhibit irregular and soft surfaces that can deform when placed on the glass slide of the MUSE sample holder, whereas FFPE tissues are rigidly embedded in wax and sectioned without 
(a) MUSE

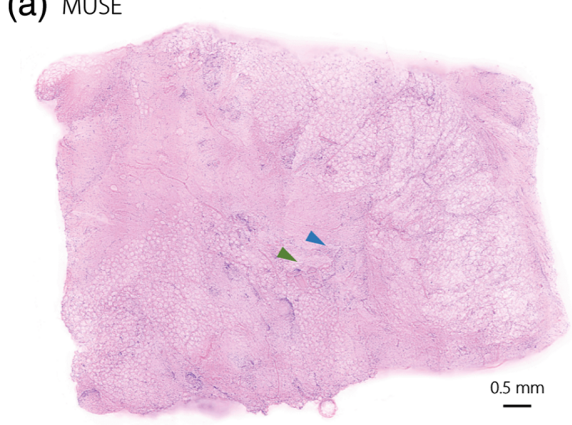

(b) Slide-based FFPE H\&E histology

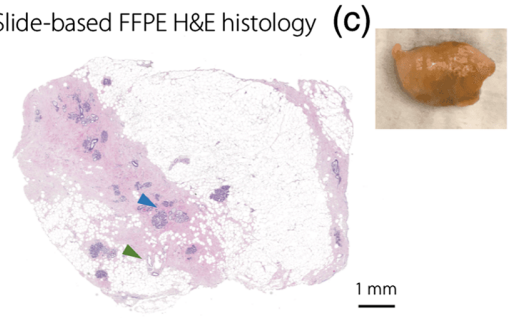

(d) MUSE

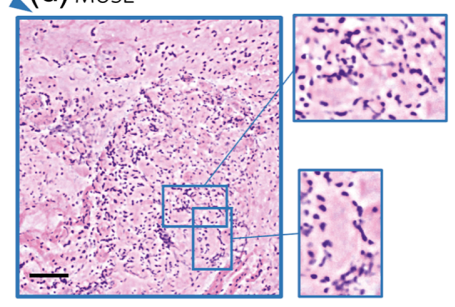

(f) MUSE

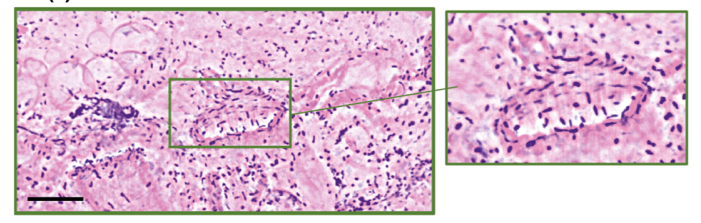

(g) Slide-based FFPE H\&E histology

(e) Slide-based FFPE H\&E histology
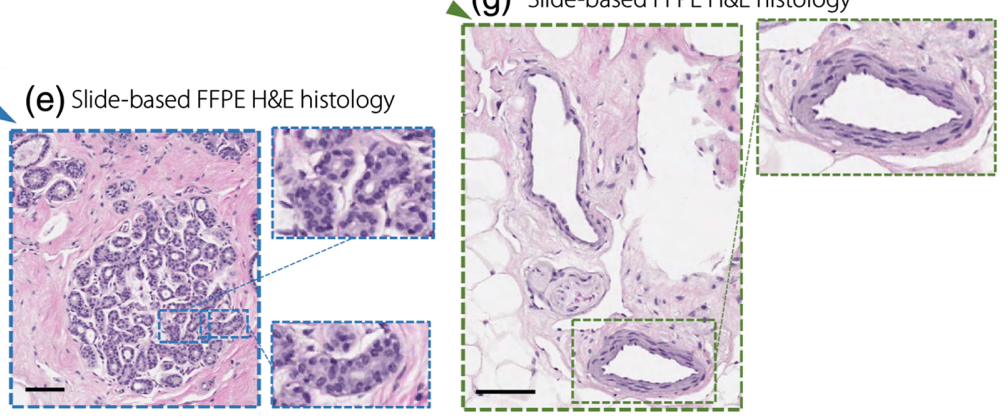

Fig. 7 MUSE imaging (H\&E analog) of benign human breast tissue. After (a) MUSE imaging, a fresh benign human breast specimen $(9 \times 10 \times 5 \mathrm{~mm})$, shown in the photo in (c), is submitted for slide-based FFPE H\&E histology (b). Images generated by (d) MUSE and (e) slide-based FFPE H\&E histology of benign breast lobules are shown with zoomed-in regions highlighting individual acini. Images generated by (f) MUSE and (g) slide-based FFPE H\&E histology of breast tissue containing collagen-rich stroma, adipose, and neurovascular bundles, with a zoomed-in region showing a venule. Scale bar: $100 \mu \mathrm{m}$ $[(d)-(g)$, first level zoom-in].

deformation. Due to the above reasons, for example, in the MUSE image shown in Fig. 8(h), a hollow structure appears to be flattened and flayed open at an angle such that the luminal surface on one side of the structure is visible. The relatively large optical-section thickness of MUSE can also contribute to this "oblique perspective," which is not seen in microtomesectioned FFPE histology.

To remove the effects of irregular surface topology while maintaining the optical-sectioning thickness of MUSE, we performed MUSE imaging of the cut surfaces of FFPE tissue blocks treated with the same SYBR-Gold-and-ATTO-655-NHS-ester staining protocol [Figs. 8(c), 8(g), 8(j), and 8(m)]. With block-face MUSE imaging, since the flat surface topology and exposed intracellular contents are similar to slide-based FFPE histology, the images [Figs. 8(c), 8(g), 8(j), and 8(m)] are more similar to standard histology than MUSE images of fresh tissues [Figs. 8(a), 8(e), 8(h), and 8(k)] are in comparison to standard histology but with some remaining differences. For example, Fig. 8(g) shows that, as with MUSE of fresh tissues, MUSE images of FFPE block faces reveal deeper layers of adipose tissue due to a thicker optical sectioning extent (10 to $20 \mu \mathrm{m}$ ) than $4-\mu \mathrm{m}$ physical sections. However, detailed ductal features in MUSE images of an FFPE block [Figs. 8(j) and 8(m)] more-closely resemble slide-based FFPE H\&E images [Figs. 8(i) and 8(1)] than MUSE images of fresh tissues [Figs. 8(h) and 8(k)] in terms of duct walls (yellow arrows) and ductal carcinoma cells (blue arrows). In summary, MUSE images of FFPE block faces support our conjecture that the differences between MUSE images of fresh tissue and slidebased FFPE H\&E images are a result of differences in both surface topology and sectioning thickness.

As a final example, Fig. 9 shows MUSE images of invasive ductal carcinoma (IDC) in comparison to slide-based FFPE
H\&E histology. Note that with similar magnification, the variable nuclear chromatin structures are more observable in slidebased FFPE H\&E images than MUSE images. We infer that this can be attributed to the two reasons stated previously: (1) differences in the optical sectioning thickness of MUSE compared with the physical sectioning thickness of slidebased FFPE histology and (2) differences in surface topology and exposed cellular structures between grossly cut fresh tissues and microtome-sectioned FFPE tissues.

While our current system can achieve imaging speeds that are relevant for intraoperative use, the MUSE system can potentially reach higher speeds to optimize its use during surgery. Currently, the imaging speed is mainly limited by optical detection sensitivity, in which an exposure time of $50 \mathrm{~ms} / \mathrm{frame}$ is required for sufficient SNR. Potential approaches to improve the imaging speed include: (1) raising the illumination power to compensate for the lower absorption cross section of the fluorescence contrast agents at $285 \mathrm{~nm}$ compared to their visiblewavelength absorption peaks. While autofluorescence background will be increased, this background is negligible at our current settings and should not be a major limiting factor. (2) Increasing the step size in the $z$ (depth) direction (the current $z$ step size is $5 \mu \mathrm{m}$ ), which will reduce the total scanning time per $z$ stack. Further experiments are needed to determine whether this would degrade the performance of our deconvolution algorithms. (3) Implementing tissue-flattening methods to reduce the topological variation of the tissue surface, such that a smaller depth range is needed to be scanned (for surface extraction). In this study, we are gently flattening the tissue against the sample holder and avoiding excessive pressures to minimize unintended artifacts. Studies are needed to determine if moreaggressive tissue-flattening mechanisms can be employed without degrading image quality. 

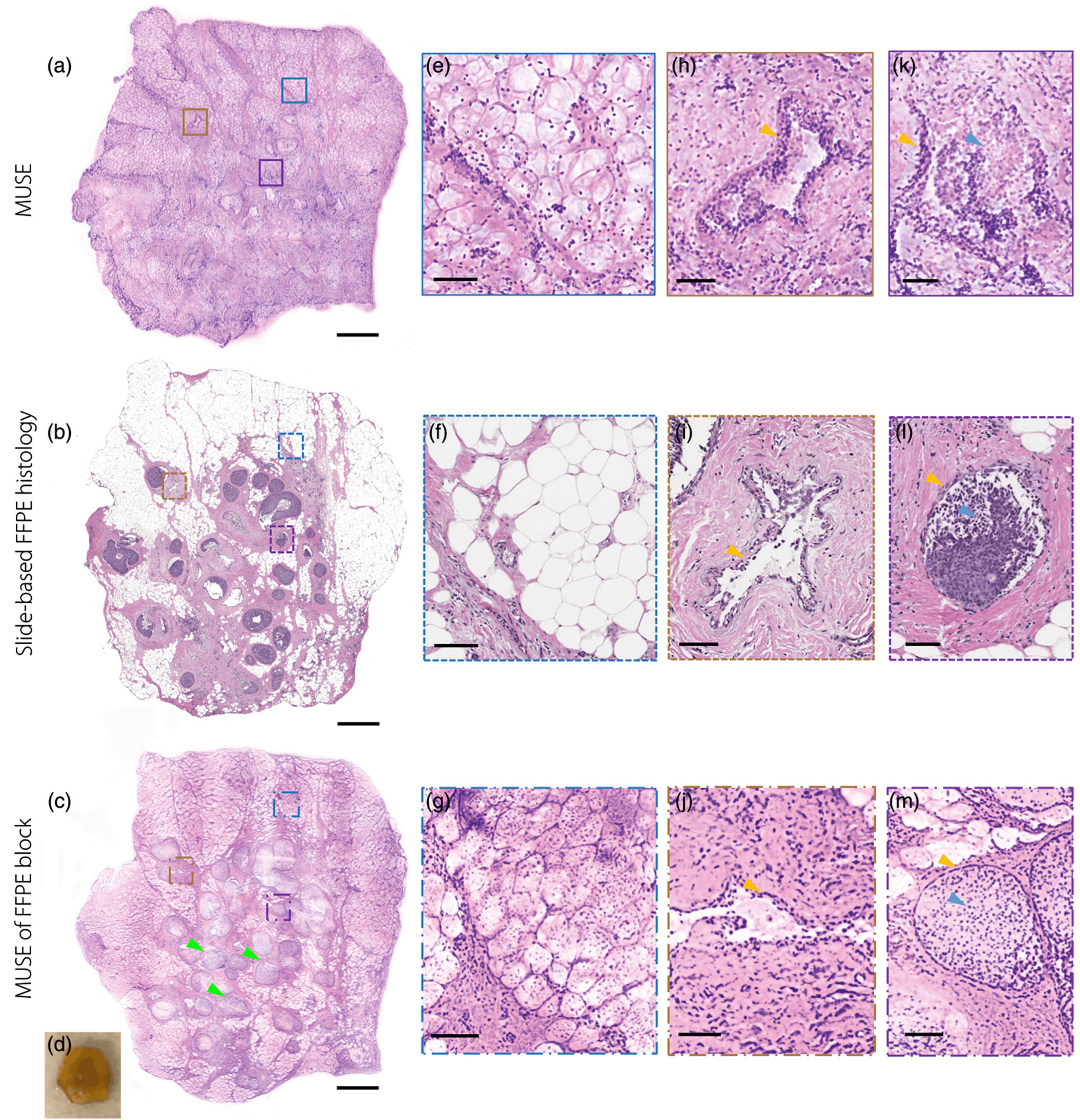

Fig. 8 Breast tissue with human DCIS (green arrows) imaged with (a) MUSE, (b) slide-based FFPE histology, and (c) MUSE of an FFPE block face. A photo of the unstained tissue is as shown in (d). Zoomed-in features imaged with (e), (h), and (k) MUSE, (f), (i), and (l) slide-based FFPE histology, and $(\mathrm{g}),(\mathrm{j})$, and $(\mathrm{m})$ MUSE of an FFPE block face. (e)-(g) Adipose tissue, (h)-(j) a benign duct, (k)-(m) DCIS. Scale bar: (a)-(c) $1 \mathrm{~mm}$ and (e)-(m) $100 \mu \mathrm{m}$.

\section{Summary}

In this study, comprehensive pathology of the margin surfaces of freshly excised breast specimens has been achieved through the use of a UV-excited two-color fluorescent analog of $\mathrm{H} \& \mathrm{E}$ in conjunction with a fully automated MUSE system that enables 3-D deconvolution and surface extraction to improve image resolution, contrast, and comprehensiveness. MUSE is a nondestructive and comprehensive surface-imaging technique, unlike slide-based FFPE histology, which requires destructive tissue sectioning with severe limitations concerning sampling extent. Image comparisons indicate that: (1) MUSE of fresh tissue provides superior image quality than frozen sectioning in that it is free from freezing and sectioning artifacts and (2) MUSE of fresh tissue provides image quality that approaches that of conventional archival $\mathrm{H} \& \mathrm{E}$ histology with certain 

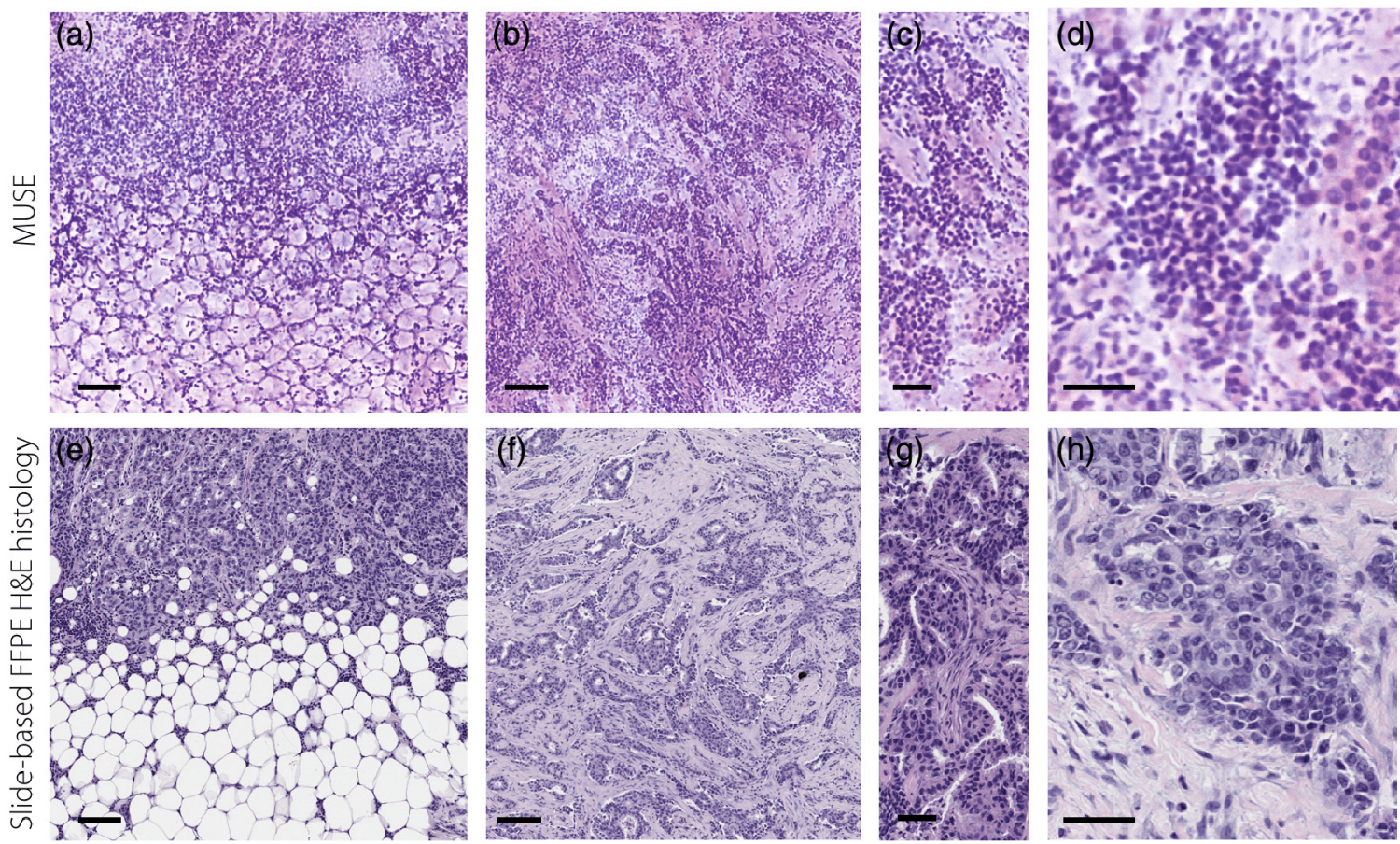

Fig. 9 Human IDC imaged with MUSE. Images generated by (a) MUSE and (e) slide-based FFPE H\&E histology are shown of IDC that has invaded a region of adipose tissue. (b) and (c) Images generated by MUSE and (f) and (g) slide-based FFPE H\&E histology of IDC that has invaded a region of fibrous stroma. Images generated by (d) MUSE and (h) slide-based FFPE H\&E histology of IDC cells with variable nuclear chromatin structure. Scale bar: (a), (b), (e), and (f) $100 \mu \mathrm{m}$ and (c), (d), (g), and (h) $50 \mu \mathrm{m}$.

disparities mainly due to differences in sectioning thickness and the topology of the cut (imaged) surfaces.

While there are apparent differences in the images generated by MUSE versus traditional slide-based FFPE histology (see Sec. 3), these differences may not ultimately be detrimental once pathologists are trained to interpret MUSE images. This work focuses on early technical advances in MUSE microscopy that support its feasibility for rapid pathology of lumpectomy margins. In the future, well-powered clinical studies, with a variety of breast pathologies, are needed to determine if the sensitivity and specificity of carcinoma detection with MUSE can compare favorably with conventional slide-based FFPE histology and to determine if the increased comprehensiveness of the MUSE imaging approach can improve detection sensitivity. In summary, this study has demonstrated the potential utility of MUSE for intraoperative surgical margin assessment to guide lumpectomy procedures, as well as other surgical oncology applications in the future.

\section{Disclosures}

No conflict of interest is declared.

\section{Acknowledgments}

Human breast tissues were provided by the NorthWest BioTrust (NWBT), which is supported in part by the NCI (No. P30CA015704 and NCI IMAT R21 CA186791). This work was funded in part by NIH Grant Nos. R01 CA175391 (Liu) and F32 CA213615 (Glaser), the University of Washington Royalty Research Fund UW RRF A107248 (Liu), a UW CoMotion Innovation Award (Liu and Reder), a Safeway/NCI SPORE developmental Award (subcontract) from the Fred Hutchinson Cancer Center (Liu and Dintzis), Prostate Cancer Foundation Young Investigator Award
(Reder), the PCRP Idea Generation Award from the Department of Defense, Congressionally Directed Medical Research Program (CDMRP) PC170176 (Liu and True), and NIDDK Diabetic Complications Consortium Grant Nos. DK076169 and DK115255 (Vaughan).

\section{References}

1. B. Fisher et al., "Twenty-year follow-up of a randomized trial comparing total mastectomy, lumpectomy, and lumpectomy plus irradiation for the treatment of invasive breast cancer," N. Engl. J. Med. 347(16), 1233-1241 (2002).

2. E. S. Hwang et al., "Survival after lumpectomy and mastectomy for early stage invasive breast cancer," Cancer 119(7), 1402-1411 (2013).

3. J. Landercasper et al., "Reasons for re-excision after lumpectomy for breast cancer: insight from the American Society of Breast Surgeons Mastery(SM) database," Ann. Surg. Oncol. 21(10), 3185-3191 (2014).

4. R. G. Pleijhuis et al., "Obtaining adequate surgical margins in breastconserving therapy for patients with early-stage breast cancer: current modalities and future directions," Ann. Surg. Oncol. 16(10), 2717-2730 (2009).

5. J. F. Waljee et al., "Effect of esthetic outcome after breast-conserving surgery on psychosocial functioning and quality of life," J. Clin. Oncol. 26(20), 3331-3337 (2008).

6. R. Jeevan et al., "Reoperation rates after breast conserving surgery for breast cancer among women in England: retrospective study of hospital episode statistics," Br. Med. J. 345, e4505 (2012).

7. T. L. Huston et al., "The influence of additional surgical margins on the total specimen volume excised and the reoperative rate after breast-conserving surgery," Am. J. Surg. 192(4), 509-512 (2006).

8. N. Cabioglu et al., "Role for intraoperative margin assessment in patients undergoing breast-conserving surgery," Ann. Surg. Oncol. 14(4), 1458-1471 (2007).

9. W. C. MacCarty, "The diagnostic reliability of frozen sections," Am. J. Pathol. 5(4), 377-380.5 (1929).

10. J. G. Whitehair et al., "The accuracy of intraoperative diagnoses based on examination of frozen-sections-a prospective comparison with paraffin-embedded sections," Vet. Surg. 22(4), 255-259 (1993). 
11. S. M. Olson, M. Hussaini, and J. S. Lewis, "Frozen section analysis of margins for head and neck tumor resections: reduction of sampling errors with a third histologic level," Mod. Pathol. 24(5), 665-670 (2011).

12. S. J. Schnitt and M. Morrow, "Should intraoperative frozen section evaluation of breast lumpectomy margins become routine practice?" Am. J. Clin. Pathol. 138(5), 635-638 (2012).

13. E. F. Brachtel et al., "Spectrally encoded confocal microscopy for diagnosing breast cancer in excision and margin specimens," Lab. Invest. 96, 459-467 (2016)

14. E. S. Flores et al., "Intraoperative imaging during Mohs surgery with reflectance confocal microscopy: initial clinical experience," J. Biomed. Opt. 20(6), 061103 (2015).

15. S. Abeytunge et al., "Confocal microscopy with strip mosaicing for rapid imaging over large areas of excised tissue," J. Biomed. Opt. 18(6), 061227 (2013).

16. Y. K. Tao et al., "Assessment of breast pathologies using nonlinear microscopy," Proc. Natl. Acad. Sci. U. S. A. 111(43), 15304-15309 (2014).

17. S. X. You et al., "Intravital imaging by simultaneous label-free autofluorescence-multiharmonic microscopy," Nat. Commun. 9, 2125 (2018).

18. M. G. Giacomelli et al., "Rapid imaging of surgical breast excisions using direct temporal sampling two photon fluorescent lifetime imaging," Biomed. Opt. Express 6(11), 4317-4325 (2015).

19. H. Tu et al., "Stain-free histopathology by programmable supercontinuum pulses," Nat. Photonics 10, 534-540 (2016).

20. M. G. Giacomelli et al., "Multiscale nonlinear microscopy and widefield white light imaging enables rapid histological imaging of surgical specimen margins," Biomed. Opt. Express 9(5), 2457-2475 (2018).

21. A. K. Glaser et al., "Light-sheet microscopy for slide-free non-destructive pathology of large clinical specimens," Nat. Biomed. Eng. 1, 0084 (2017).

22. T. C. Schlichenmeyer et al., "Video-rate structured illumination microscopy for high-throughput imaging of large tissue areas," Biomed. Opt. Express 5(2), 366-377 (2014).

23. M. Wang et al., "Gigapixel surface imaging of radical prostatectomy specimens for comprehensive detection of cancer-positive surgical margins using structured illumination microscopy," Sci. Rep. 6, 27419 (2016).

24. M. Wang et al., "High-resolution rapid diagnostic imaging of whole prostate biopsies using video-rate fluorescence structured illumination microscopy," Cancer Res. 75(19), 4032-4041 (2015).

25. T. T. W. Wong et al., "Fast label-free multilayered histology-like imaging of human breast cancer by photoacoustic microscopy," Sci. Adv 3(5), e1602168 (2017).

26. O. M. Carrasco-Zevallos et al., "Review of intraoperative optical coherence tomography: technology and applications [Invited]," Biomed. Opt. Express 8(3), 1607-1637 (2017).
27. S. J. Erickson-Bhatt et al., "Real-time imaging of the resection bed using a handheld probe to reduce incidence of microscopic positive margins in cancer surgery," Cancer Res. 75(18), 3706-3712 (2015).

28. F. Fereidouni et al., "Microscopy with ultraviolet surface excitation for rapid slide-free histology," Nat. Biomed. Eng. 1, 957-966 (2017).

29. T. Yoshitake et al., "Rapid histopathological imaging of skin and breast cancer surgical specimens using immersion microscopy with ultraviolet surface excitation," Sci. Rep. 8, 4476 (2018).

30. A. Qorbani et al., "Microscopy with ultraviolet surface excitation (MUSE): a novel approach to real-time inexpensive slide-free dermatopathology," J. Cutaneous Pathol. 45(7), 498-503 (2018).

31. R. Zanella et al., "Towards real-time image deconvolution: application to confocal and STED microscopy," Sci. Rep. 3, 2523 (2013).

32. M. A. Bruce and M. J. Butte, "Real-time GPU-based 3D deconvolution," Opt. Express 21(4), 4766-4773 (2013).

33. K. N. Elfer et al., "DRAQ5 and Eosin ('D\&E') as an analog to hematoxylin and eosin for rapid fluorescence histology of fresh tissues," PLoS One 11(10), e0165530 (2016).

34. R. S. Tuma et al., "Characterization of SYBR gold nucleic acid gel stain: a dye optimized for use with 300-nm ultraviolet transilluminators," Anal. Biochem. 268(2), 278-288 (1999).

35. B. Forster et al., "Complex wavelets for extended depth-of-field: a new method for the fusion of multichannel microscopy images," Microsc. Res. Tech. 65(1-2), 33-42 (2004).

36. S. Preibisch, S. Saalfeld, and P. Tomancak, "Globally optimal stitching of tiled 3D microscopic image acquisitions," Bioinformatics 25(11), 1463-1465 (2009).

37. M. G. Giacomelli et al., "Virtual hematoxylin and eosin transillumination microscopy using epi-fluorescence imaging," PLoS One 11(8), e0159337 (2016).

Weisi Xie received her BEng degree in optical engineering and BEng degree in industrial design from Zhejiang University, China, in 2017. She is a current PhD student studying in the Mechanical Engineering Department at the University of Washington, Seattle, Washington, USA. Her research interests include microscopy, cancer detection, and machine learning for biomedical imaging applications.

Jonathan T. C. Liu received his BSE from Princeton, and his $\mathrm{PhD}$ from Stanford in 2005. He was postdoc in the molecular imaging program at stanford and then became an assistant professor at Stony Brook from 2010-2014. Currently, he is an associate professor in mechanical engineering at the University of Washington, with an adjunct appointment in pathology. He Molecular Biophotonics Lab develops optical strategies to improve the diagnosis and treatment of diseases.

Biographies of the other authors are not available. 